\title{
Sujeto y subjetividad: la problemática de las alternativas como construcción posible
}

\author{
Hugo Zemelman Merino \\ IPECAL, México D.F., México. Email: ipecal_mexico@yahoo.es
}

\begin{abstract}
Resumen: El artículo aborda el desafío de una conceptualización de la realidad socio-histórica que rompa con la separación entre lo real como externalidad y el sujeto. Lo anterior pasa por redefinir la idea de objetividad. Se requiere encontrar un concepto de subjetividad constituyente que no sea operativo por reducciones al plano de las variables psicológicas, pero que tampoco se resuelva como simple expresión de procesos macrohistóricos. Desde un punto de vista metodológico, realza el contrapunto entre contenidos teóricos permanentes y la transitoriedad de los sujetos. Se afirma que para poder armonizar desarrollo humano y desarrollo social, se requieren relaciones sociales que no impliquen dominación económica ni política. Llegado a esto, se pregunta: ¿Es que el capitalismo se esconde detrás de la democracia, por lo que ésta, en su propio nombre, se niega así misma?
\end{abstract}

Palabras clave: objetividad, externalidad, sujeto, desarrollo humano, procesos macrohistóricos

\section{Subject and subjectivity: the challenge of alternatives as a possible setting-up}

\begin{abstract}
This article addresses the challenge of a conceptualization of the socio-historical reality that can end the separation between the reality as externality and the subject. The aforesaid requires a redefinition of the idea of objectivity. There is the need to find a concept of subjectivity that wont operate by reduction to the level of psychological variables, nor as a simple expression of macrohistorical processes. From a methodological perspective, it highlights the ongoing counterpoint between theoretical content and transience of the subjects. It states that in order to attune human development and social development, social relationships are required that wont involve economic or political domination. Come to this, it is asked: Is it that capitalism hides behind democracy, so this, in its own behalf, denies herself?
\end{abstract} macrohistorical

Key words: objectivity, externality, subject, human development, processes

\section{Sujeito e subjetividade: o desafio da construção de alternativas possível}

Resumo: O presente artigo aborda o desafio de uma conceituação da ruptura sócio-histórica com a separação entre a realidade ea exterioridade eo sujeito. 
Isso acontece para redefinir a ideia de objectividade. Ele iria procurar um conceito constitutivo de subjetividade que não está operando, através de reduções ao nível das variáveis psicológicas, mas também não é resolvido como um simples expressão do processo macrohistorical. Do ponto de vista metodológico, destaca o contraponto permanente entre conteúdos teóricos e transitoriedade dos indivíduos. Afirma que, a fim de levar o desenvolvimento humano eo desenvolvimento social, as relações sociais são necessários que não envolvem a dominação econômica ou política. Vamos a isso, ele se pergunta: Será que o capitalismo está por trás da democracia, de modo que este, em seu próprio nome, negou-se?

Palavras-chave: objetividade, externalidade, desenvolvimento, sujeitos humanos, os processos macrohistorical

\section{Consideraciones preliminares}

El problema de los sujetos sociales no puede desvincularse de las cuestiones básicas del conocimiento social. Por una parte, por las circunstancias de que ninguna realidad social concreta puede entenderse sin la presencia de algún tipo de sujeto; y de otra, que, a pesar de la importancia de éstos, enfrentamos graves dificultades para comprenderlos en toda su complejidad.

El primer desafío se traduce en tener que problematizar lo que se entiende por realidad socio-histórica, en forma de llegar a una conceptualización de ésta que rompa con la separación entre lo real como externalidad y el sujeto. Lo anterior significa redefinir la idea de objetividad de manera de encontrar un concepto más congruente de ésta, como puede ser la idea de espacios de posibilidades en los que tienen lugar la existencia de los sujetos y el consiguiente despliegue de sus capacidades de construcción.

Desde esta perspectiva, debemos enfrentar el desafío de comprender en que consiste la capacidad de construcción que debemos abordar en toda su complejidad: abordar lo que significa construir, en tanto capacidad de los sujetos, pero también comprender el significado social de lo que representa lo construido.

No es posible pensar en ningún tipo de estructura social, económica o política, como tampoco cultural, si no es como resultado de la presencia de sujetos en complejas relaciones recíprocas en cuanto a tiempos y espacios; lo que implica tener que enfocar los procesos como construcciones que se van dando al compás de la capacidad de despliegue de los sujetos, los cuales establecen entre sí relaciones de dependencia recíproca según el contexto histórico concreto.

Pero reenfocar la realidad desde las exigencias de estos espacios de posibilidades significa romper con el condicionamiento de lo invariante para poder rescatar el movimiento interno de lo dado, que es el movimiento de los sujetos, que plasma la dinámica interna de lo dado. De ahí que para 
leer la realidad debemos saber leer el movimiento constituyente de los sujetos, lo que tropieza con diferentes obstáculos en general asociados a formas de pensar que rehuyen pensar simplemente lo que carece de formas claramente definidas.

En este sentido, surgen diferentes tópicos desde esta exigencia de pensar la realidad conformada por espacios de despliegue, pues lo que llamamos dinámicas sociales son una manifestación de estos despliegues y de cómo se pueden transformar en diferentes tipos de capacidades de construcción.

La primera consideración refiere a la necesidad de estar alertas acerca de que, desde esta perspectiva, las estructuras de la realidad socio-histórica pueden revestir significados diferentes según los sujetos, ya que pueden tener sentido para unos pero no para otros, ello debido a que pueden representar espacios para sus despliegues, a la vez que no serlo para otros sujetos. Por ejemplo, está claro que las instituciones económicas no significan lo mismo para diferentes sujetos, como ser los bancos, las bolsas de valores, etc., como puede también ocurrir que las instituciones políticas tampoco revistan el mismo significado para todos los sujetos, en cuanto constituir posibilidades de despliegue.

En esta dirección, el estado puede conformar un espacio de participación directa para los grupos dominantes, mientras que para los subordinados puede plantear la presencia de mediaciones, a veces tan complejas e ineficaces que no tienen un significado efectivo para muchos sujetos. De ahí que la primera cuestión que se tiene que plantear como eje ordenador del pensamiento, es lo que concierne al orden en cuanto este conforma un mecanismo constitutivo de subjetividades, mediante sus múltiples recursos, al influir sobre el sistema de necesidades de los colectivos sociales.

Pero lo que decimos es expresión del hecho de que los sujetos son siempre sujetos situados en relaciones múltiples y heterogéneas, las cuales conforman el espacio que los determina en la naturaleza de su movimiento, que se traduce, en primer lugar, en el surgimiento de la necesidad de ocupar un espacio en el que tiene lugar el reconocimiento a pertenencias colectivas, lo que se acompaña de la conformación de una subjetividad social particular. Lo que apunta a la problemática de la aparición de los colectivos con sus respectivas amplitudes tiempo-espaciales, así como en una densidad propia.

El sujeto deviene en una subjetividad constituyente, en la medida que requiere entenderse en términos de cómo se concretiza en distintos momentos históricos; de ahí que al abordar a la subjetividad como dinámica constituyente, el sujeto es siempre un campo problemático antes que un objeto claramente definido, pues desafía analizarlo en función de las potencialidades y modalidades de su desenvolvimiento temporal. Por eso su abordaje tiene que consistir en desentrañar los mecanismos de esta subjetividad constituyente, tanto como aclarar los alcances que tiene la subjetivi- 
dad constituyente. Plantea distinguir entre producto histórico y producente de nuevas realidades.

En cuanto expresión de la subjetividad social constituyente, el sujeto se tiene que observar como proceso que se puede especificar, en lo que respecta a sus contenidos, en distintos recortes de observación; en otras palabras, según diferentes parámetros, por lo que la observación no se puede agotar en un solo momento, ya que su "objetividad" consiste en una diversidad de modos de concreción de la subjetividad; lo que obliga a considerar la relación entre momento y secuencia, por eso el riesgo de manejar un concepto a priori. Más bien se tiene que considerar que puede reconocer distintos planos para manifestarse, tal como pueden serlo los propios de la cotidianidad que se muestra en la situación de vida y de trabajo; o bien, los planos en los que se manifiesta la relación memoria-utopía y el propio sistema de necesidades.

Por lo anterior, es importante recuperar el concepto de ritmo de constitución, pues serán esas diferencias las que marquen la distinción entre el micro y el macroproceso social. En este sentido es sugerente trabajar metodológicamente las distinciones temporales hechas por Braudel y establecer un vínculo con la idea gramsciana de movimiento molecular. La principal dificultad reside en resolver lo que refiere a las dinámicas constitutivas, en razón a la tendencia a reducirlas a mecanismos propios de la subjetividad individual; o, en su defecto, en una reconstrucción de las condiciones “externas” que, eventualmente, la determinan.

La problemática central se encuentra en no manejar un concepto de determinación que no considere las mediaciones entre estas condiciones y la emergencia de subjetividad, que no necesariamente se somete a una evolución progresiva. El desafío está en encontrar un concepto de subjetividad constituyente que no sea operativo por reducciones al plano de las variables psicológicas, como tampoco que se resuelva como simple expresión de procesos macrohistóricos.

Si la subjetividad es un campo problemático que conjuga las dimensiones micro y macrosociales, supone tener que reconocer la dialéctica que, pudiendo darse en un plano de la realidad, sea productora de realidades incluyentes. La necesidad y la experiencia articulan lo micro con lo macrosocial, pero en su reproducción se proyecta en microplanos, así como es posible que en su concreción reflejen dinámicas macrosociales.

Desde este ángulo, se hace necesario profundizar en los mecanismos constitutivos de la subjetividad, para no congelar la problemática en una tipología de conductas. A este respecto, se pueden destacar momentos del proceso de la subjetividad social, comenzando por aquellos que pueden ser básicos de la subjetividad, de conformidad con un enfoque no psicologista, a saber el mundo de las necesidades, sin caer en el particularismo de ninguna demanda concreta. Mundo constituido por dos génesis de necesidades: la memoria (tradición, inercia), y, de otra parte, las 
visiones de futuro, la utopía de algo. El punto de tensión entre estas dos grandes polaridades delimita el primer ámbito de las posibles necesidades.

A este momento, propio de la dialéctica memoria-utopía, sigue otro que se vincula con el reconocimiento de opciones con base en el desarrollo de la capacidad para construir proyectos. En este caso, el sujeto representa una potencialidad realizada en términos de determinadas alternativas de sentido: esto es, pasa de la pura potencialidad, propia del primer momento, que contiene múltiples posibilidades de sentido, a la concreción de una alternativa particular de sentido.

Pero este momento puede estar fuertemente moldeado por factores externos (ideologías, exigencias políticas concretas, distorsiones organizativas, etcétera) que alteren su dinámica interna. Es posible que se impongan determinadas interpretaciones de la realidad, las que pueden moldear un deseo de futuro que se imponen a lo que hay de potencial en el sujeto. En esta situación, la utopía se convierte en una meta externa al movimiento constitutivo de la subjetividad, respondiendo, más bien, a una ideología acerca de lo que significa trascender la realidad dada, en vez de ser un mecanismo de reconocimiento de la potencialidad que se contiene en dicha situación dada.

En este caso, nos encontramos en un momento del proceso de constitución de la subjetividad que es propio del sujeto movilizado, que puede distorsionar sus efectivas potencialidades internas, ya que la subjetividad dependerá de lo que pretenda hacer con el sujeto un agente externo. Su transformación en fuerza se hace con base en la construcción de un proyecto que es impuesto, sin que medie el desenvolvimiento de su capacidad para reconocer opciones y de reconocer su viabilidad.

\section{Situación histórica}

En esta dirección, es importante reconocer las situaciones históricoconcretas que distintos sujetos pueden crear para presentarse como portadores de prácticas que sean congruentes con determinadas visiones del mundo social. Pero que, a su vez, plantea que el mismo sujeto puede conllevar una impronta de naturaleza coyuntural que difícilmente supere.

Una posibilidad es que un proceso electoral, determine el surgimiento de un movimiento, con organización amplia y sólida, con discurso y proyecto, pero marcada la organización, así como las visiones, por la coyuntura que contribuye a constituirlo, en este caso, un triunfo electoral. Lo dicho implica que "esa fuerza electoral" del sujeto no sea suficiente para permitirle avanzar en la profundización de su proyecto planteándose la necesidad de transformar la naturaleza de la organización, sea partidista o de otra índole, con la que ha surgido el sujeto en la coyuntura, y que nos plantee la relación entre sujeto y actores políticos. 
En efecto, una lógica clientelística propia de confrontaciones electorales puede no ser la adecuada para una lucha orientada a enfrentar al contrincante en sus contradicciones internas, como ser dividir al bloque burgués, ya que hacerlo supone el diseño de políticas de una duración prolongada como la que exige una lucha contra-hegemónica. De lo que se infiere que no sea suficiente provocar polarizaciones y alienamientos entre fuerzas a partir de prácticas que expresan expectativas y demandas inmediatas, a veces, contingentes y de poca influencia, cuando el proyecto requiere de un tiempo prolongado para afianzarse.

La cuestión a la que hacemos referencia se puede ilustrar con lo que ocurrió con la política económica de la Unidad Popular en Chile (Gobierno de Salvador Allende 1970-1973) frente a las capas medias, orientadas a darles a éstas una mayor capacidad de consumo, con la expectativa de crear una alianza con la Unidad Popular. Ello no ocurrió en la medida que se trataba de un proyecto contrahegemónico, que, en la medida que no llegó a romper con la dependencia clientelística, no pudo recomponer el espacio en el que se configuraba la hegemonía del poder dominante.

El problema consistió en que no se pudo pasar de una dinámica clientelar de naturaleza electoral a la de un movimiento que se proyectaba en el largo tiempo, exactamente en torno de una nueva concepción de sociedad, más allá de las alianzas programáticas de corto plazo. De hecho, se estaba dando una transformación de la organización misma que sustentaba al proyecto (la alianza entre partidos políticos, y con ello el significado de las representaciones). La cuestión se ha prestado a constantes discusiones en torno de cómo se puede manifestar la voluntad colectiva cuando se pasa de una coyuntura a otra en la que se transforma la naturaleza de las fuerzas, y, en consecuencia, la misma correlación entre éstas.

En efecto, en un momento se puede dar la emergencia de una alianza con muchos sujetos alrededor de un proyecto compartido: Por ejemplo, la lucha por la democracia, o el interés de desplazar del poder institucionalizado a un determinado sector social, pero que una vez logrado el objetivo puede tener significados diferentes para los distintos sujetos. Aunque lo más relevante tiene lugar cuando el propio sujeto, se queda prisionero de una determinada coyuntura hasta el punto de sesgar su lectura de sociedad al circunscribirse a la lógica particular de la coyuntura que conforma la organización y la ideología del sujeto.

Puede ser el caso de sujetos que se quedan fijados por una estrategia militar sin saber como pasar a una etapa diferente en lo que se refiere a organización y conciencia, de manera de definir tácticas de luchas diferentes. O, a la inversa, que han sido marcados por el éxito en la sociedad civil, como resultado de formas de lucha que se dan en el marco de la institucionalidad, pero que al cambiar la correlación de fuerza, y no ser capaz de asumir el cambio, puede perder cohesión ideológica y efectividad política. 
La primera situación se puede ilustrar con lo que ocurrió con la Unidad Nacional Revolucionaria Guatemalteca (UNRG), después de los acuerdos de paz, mientras que la segunda lo ilustra la Unidad Popular de Chile, caracterizada por ser una alianza entre partidos políticos exitosos electoralmente, pero que no fueron capaces de enfrentar un cambio en el espacio de la correlación de fuerzas. En el primer caso el no paso de lo militar a lo civil, en el segundo, el no paso de lo civil a lo militar.

\section{Desafíos metodológicos}

Desde esta visión, la constitución de la subjetividad plantea tener que reconocer momentos, que, teóricamente, son claros pero que no son fáciles de analizar metodológicamente, como ser: i) el momento de coaligarse en el plano individual y/o primario, ii) la constitución de referentes de pertenencia más incluyentes, iii) la consolidación de disociaciones sociales entre realidades estables y su expresión en prácticas sociales, y iv) desde éstas a las formas de organización que la potencian y garanticen su proyección.

La cuestión que subyace remite a la conformación de la capacidad de construcción que no se puede separar de la amplitud del despliegue, pues si el despliegue está restringido a determinadas situaciones sociales nunca tendrá la fuerza de un eventual sujeto. Es el desafío que enfrentan, a manera de ilustración, los sindicatos que al restringirse a lo puramente gremial pueden perder fuerza en un momento histórico en el que se requiera que se transformen en movimientos sociales.

En esta dirección, es importante destacar la función inhibitoria que pueden tener ciertas formas de organización sobre la constitución de la subjetividad cuando la aprisionan en modelos que sirven para canalizarla para determinados propósitos, muchas veces puramente clientelísticos. Discusión que ha sido permanente a lo largo de todo el siglo XX, ya que a través de las formas de organización se consolida un vinculo del movimiento de la subjetividad con el orden institucionalizado. De ahí la importancia de reconocer, en los límites de las determinaciones sociales, márgenes de actuación de los sujetos, lo que supone saber enfocar el tránsito desde lo constitutivo a lo establecido como estructura, organización o institución. Desde un punto de vista metodológico, lo que decimos plantea no reducir el análisis de los sujetos a sus cristalizaciones históricas.

Se puede reconocer la presencia de la concepción gramsciana de la política, en cuanto no restringirse al dispositivo central del estado, sino, más bien, abordar la problemática de la subjetividad social, con posibilidades diferentes de densidad, en la acepción de Canetti, que plantea abordar la cuestión de la articulación de espacios que pueden moverse desde los modos de vida, el plano de las formas de organización social como el de los vínculos interpersonales, incluyendo los ámbitos propios de los mundos simbólicos, con sus correspondientes prácticas, hasta las subculturas socia- 
les y los espacios propios de los mecanismos centrales del dominio político propios del estado. De ahí que no se puedan comprender las dinámicas constitutivas de la subjetividad social si no se considera el análisis de la actuación de la gente corriente.

En efecto, se tiene que resolver el movimiento interno del momento que exige superar las formas de razonamiento ceñidas a los requerimientos de explicación, los cuales contribuyen a hacer de la relación del sujeto con sus circunstancias una externalidad, respecto del propio sujeto, dejando fuera la exigencia central de un pensamiento histórico concreto que refiere a la dimensión de la constitución de lo real, a partir de lo potencial que se contiene en los márgenes de construcciones posibles de los sujetos.

Por estas razones, se platea una ampliación en la relación de conocimiento de modo que sea congruente en la incorporación del sujeto en sus circunstancias, lo que obliga a concebir al conocimiento como parte de una relación más incluyente, que hace difícil separarla de opciones valóricas con base en las cuales tiene lugar la construcción de sentido. La realidad deviene en una constante construcción de sentidos.

De lo anterior se derivan algunos rompimientos respecto de la tendencia teórico-explicativa, en la medida en que se enfatiza como exigencia central reconocer el sentido que tiene el conocimiento cuando se concibe como parte de un esfuerzo de construcción (que, además, tiene lugar en coordenadas de tiempo y espacio de distinta amplitud y estabilidad). En consecuencia, surge la necesidad de dar cuenta de lo que significa hablar de contenidos teóricos permanentes y de la función que cumple reconocer opciones desde la transitoriedad de los sujetos. En efecto, ¿ en qué consiste conocerlos? ¿son objetos verdaderamente teorizables?, o bien, ¿qué se entiende en este contexto por teorizar?

No podemos desconocer que la problemática de los sujetos y de su conocimiento incluye cuestiones como las que implica enfrentarse con el modo de leer el sistema de necesidades y las prácticas orientadas a satisfacerlas, así como la presencia de mediaciones constitutivas de visiones y de vivencias, de modo de no perder su relación con las circunstancias que configuran la lógica del orden; desafío que no se ajusta necesariamente con formulas pulidas y equilibradas, propias de las lógicas cognitivas y asertóricas.

Nos colocamos ante una exigencia de conocimiento que supone concebir la realidad como espacio donde se construyen sentidos, lo que obliga a incorporar el estar-siendo del sujeto, así como sus relaciones con otros. Es lo que obliga a comprender a los procesos sociales desde la recuperación de sus dinámicas gestantes, ocultas detrás de todas las formas establecidas y que se desplazan en diversos tiempos y espacios; dinámica entre las que caben destacar la necesidad de ser sujeto que, aunque callada, nos constituye. 
De ahí que haya que enfocar al sujeto desde sus límites pero sin reducirlo a sus determinaciones históricas; en consecuencia, pensar los límites de los conceptos en forma de transgredirlos para convertirlos en posibilidades de renovados contenidos, según sea la capacidad de construcción de los propios sujetos sociales. Este es uno de los principales desafíos para comprender el surgimiento, desarrollo, transformación y desaparición de los sujetos sociales, en forma de no incurrir en el manejo de "conceptos cadáveres” (Adorno) que aludan a “documentos muertos” (Gramsci).

Por lo que decimos, es necesario abandonar la óptica de análisis centrada en la relación sujeto-objeto, ya que puede en aras de pretensiones teóricas de universalidad conllevar la idea de una objetividad externa posible de apropiarse en un corpus relativamente permanente, en circunstancias que se trata de abordar la construcción del conocimiento para dar cuenta de momentos históricos delimitados y en secuencia temporal, incluyente de múltiples relaciones, en los que tiene lugar el despliegue de los sujetos.

Lo que decimos implica definir en el centro del debate la cuestión de los contenidos, y, en consecuencia, de los instrumentos necesarios para construirlos, en forma de garantizar que reflejen el movimiento de lo dado, esto es, el despliegue de los sujetos, que a veces puede ocultar la descripción de la realidad entendida como objetivación. Es lo que pone en lugar epistémico privilegiado a las categorías para responder a los desafíos de conocimiento que no se circunscriben estrictamente a exigencias de legaliformidad.

En lo expuesto subyace la idea de la necesidad de ser sujeto, que, desde nuestro punto de vista, no plantea cuestiones ontológicas, pues más bien refiere al status de la historicidad en tanto ángulo de razonamiento. Se relaciona con la aceptación del carácter inacabado del sujeto, con su misma indeterminación, como consecuencia del movimiento de lo real, por lo tanto del mismo sujeto. El desafío de la historicidad se expresa en asomarse a lo desconocido; por eso, la principal tensión en el análisis de los sujetos esté representada por la presencia de visiones de futuro, ideológicas o escatológicas, que no pueden perturbar con su presencia la exigencia de distanciamiento crítico en el análisis de los sujetos, ya sea que estén emergiendo o estén desarrollándose. Es el desafío ético y metodológico, estrechamente vinculados, que caracteriza el esfuerzo científico por dar cuenta de lo desconocido de la historia.

Sin embargo, asomarse a lo desconocido requiere atreverse a asumirse como sujetos pensantes desde el conjunto de sus facultades. Expresa el esfuerzo por colocarse en lo dado desde su mismo transcurrir. De alguna manera se corresponde con el desarrollo humano en tanto constante ampliación de la subjetividad como fuerza modeladora de la sociedad.

Pero para poder armonizar el desarrollo humano con el desarrollo social, se requiere organizar la sociedad con base en relaciones sociales que no impliquen dominación económica ni política, a pesar de saber que 
en toda sociedad ha sido la división del trabajo la que ha servido de fundamento para estructurar la dominación. Y desde ésta la organización del propio pensamiento, por lo que el análisis de los sujetos como forjadores de realidad, que trascienden los parámetros del poder, requiere de un esfuerzo máximo de distanciamiento de los sesgos que impone la lógica de dominación como lógica que impone una mirada de lo social. De lo que se desprende la importancia de una forma de pensar la realidad histórica desde la potencialidad que se contiene en el surgimiento y transformación de los sujetos, con toda la fuerza que contienen, más allá de las formas organizativas que asuman en un momento histórico dado.

\section{Problemática sociopolítica en América Latina}

Como consecuencia de lo que venimos argumentando, no se puede dejar de reconocer la dificultad que representa vislumbrar futuros en una situación, como la del capitalismo, que resignifica una variedad de fenómenos que nos lleva a pensar en una situación inestable, abierta en su dirección a un devenir incierto pero que el poder pretende mostrarla como cerrada, sin alternativas. De ahí que la tarea esencial sea procurar construir los lineamientos principales de este momento caracterizado por la crisis del modelo neoliberal, que no es sino manifestación de los desequilibrios estructurales del capitalismo y la consiguiente emergencia de proyectos políticos que lo cuestionan. Es el caso de Cuba, y ahora el de Venezuela, Bolivia y Ecuador, como las experiencias más recientes.

Debemos partir reconociendo que la situación se define por la alta concentración del capital y el predominio sin contrapeso del capital financiero, lo que da lugar a un crecimiento con precariedad del empleo e insuficiencia de ingresos, determinando que grandes sectores sociales estén excluidos y carezcan de perspectivas de futuro.

Crisis económica-financiera que se produce, además, en el contexto político caracterizado por un estado debilitado, sin los instrumentos de regulación como resultado del predominio de una lógica de mercado. Economía de mercado que se acompaña de posturas que enfatiza la eficacia aunque cortoplacistas, dando lugar a un fuerte individualismo que mutila las posibilidades de que surjan colectivos; individualismo que más allá de su conformidad permanece inerme en sus situaciones de desventaja. El mismo concepto de futuro se reduce a la idea de oportunidades intervinientes, a una capacidad del sujeto que se reduce a comportamientos de movilidad social individual.

De manera que se crean las condiciones para disolver cualquier referente de pertenencia colectiva, por lo que el significado de lo social se disuelve en una atomización que es el caldo de cultivo de la competitividad y del reconocimiento del éxito personal como mecanismo para mostrar presencia social. La sociedad se subsume en la movilidad individual, mientras los horizontes históricos se restringen a los límites de proyectos personales 
de vida, en tanto que la solidaridad no se plasma en ninguna identidad colectiva, sino, en el mejor de los casos, en acciones de solidaridad en relación con situaciones de marginalidad o de violencia de los derechos humanos; aunque quizá, su forma dominante, se encuentre en la lógica de cooptación impulsada por grupos particulares. La solidaridad, en definitiva, como expresión de asistencia social que es como funcionan los sindicatos pero también los partidos.

Las consecuencias de lo que decimos son profundas sobre las instituciones y los espacios en los que se puede ejercer el poder. El capitalismo no está negando a la democracia, pero sí la está transformando de conformidad a sus propias necesidades. Cada vez más la marginación de sectores, así como la pérdida de proyectos, la inestabilidad laboral o la precariedad de ingresos, agudizan sus efectos, lo que plantean la necesidad de políticas de control, que a su vez, muestran la apariencia de apertura a la participación que facilitan que el poder no pierda su hegemonía.

Entre estos mecanismos se pueden señalar la exaltación por hacer ganar presencia a la sociedad civil como espacio de participación y de poder, pero que no es sino un montaje para disfrazar la presencia de los poderes fácticos; los cuales, la debilitan en un juego que aparece favoreciendo las libertades cívicas, como ser la participación ciudadana, o como apoyarse en los "emprendimientos sociales", pero que ocultan el predominio sin contrapeso de estos poderes.

La institucionalidad del estado se reduce a un espacio en el que se persigue una suerte de arbitraje entre las fuerzas sociales, de modo que termina por cumplir la función de asistencia social para controlar las emergencias de proyectos alternativos; aunque estos últimos no requieran de la presencia de un "estado en forma". En verdad, del espacio de las instituciones se han apoderado los poderes fácticos económico-financieros así como los grupos de presión político de carácter clientelística. Ello lleva a que la democracia esté deviniendo en el sistema político que legitima las dinámicas económicas y políticas que operan fuera de las instituciones: la democracia como manifestación del estado de excepción.

En razón de las consideraciones anteriores, debemos destacar el carácter problemático de la relación entre proyecto político y económico, en la medida que se plantee la cuestión de si es posible construir un modelo económico no capitalista desde la democracia; o bien, si el modelo económico se protege con la democracia ante la voluntad de construcción de proyectos económicos alternativos. En otras palabras, los liderazgos que hoy impulsan otras estrategias ¿podrán mantenerse en la democracia representativa clásica, o, más bien, están compelidos a construir otros sistemas políticos de participación ciudadana? ¿Acaso la democracia necesariamente impone, aunque con variantes, un proyecto capitalista? ¿Es que el capitalismo se esconde detrás de la democracia, por lo que ésta, en su propio nombre, se niega así misma? ¿Es el capitalismo el sistema económico que requiere de la democracia para legitimarse, pero que en esta función repre- 
senta la negación de la democracia en nombre de la democracia? ¿No es lo que decimos la máxima expresión de lo que es la naturaleza más profunda de la hegemonía?

Recibido: 22.10 .2010

Aceptado: 17.11.2010 